\title{
Causal Inference on Pathophysiological Mediators in Psychiatry
}

\author{
Ho Namkung, ${ }^{1,2}$ Brian J. Lee, ${ }^{2}$ And AkiRa SAwA ${ }^{1,2,3,4}$ \\ ${ }^{1}$ Department of Biomedical Engineering, \\ ${ }^{2}$ Department of Psychiatry and Behavioral Sciences, \\ ${ }^{3}$ Department of Neuroscience, Johns Hopkins University School of Medicine, Baltimore, \\ Maryland 21287, USA \\ ${ }^{4}$ Department of Mental Health, Johns Hopkins University Bloomberg School of Public Health, \\ Maryland 21287, USA \\ Correspondence: asawa1@jhmi.edu
}

\begin{abstract}
Supported by technological advances and collaborative efforts, psychiatric genetics has provided robust genetic findings in the past decade, particularly through genome-wide association studies (GWASs). However, translating these genetic findings into biological mechanisms and new therapies has been enormously challenging because of the complexity of their interpretation. Furthermore, the heterogeneity among patients with the same diagnosis, such as schizophrenia or major depressive disorder, challenges the biological validity of existing categorical approaches in clinical nosology, which is further complicated by the pleiotropic nature of many genetic variants across multiple disorders. Therefore, in the post-GWAS era, the greatest challenge lies in integrating such enriched genetic information with functional dimensions of neurobiological measures and observable behaviors. In this integration, the causal inference from genotypes to phenotypes through intermediate biological processes is of particular importance. In this review, we aim to construct an intellectual framework in which we may obtain causal, mechanistic insights into how multifactorial etiologies — in particular, many genetic variants - affect downstream biological pathways that lead to dimensions of psychiatric relevance.
\end{abstract}

Neuropsychiatric disorders place an enormous burden on affected individuals, their families, and society. They are the leading cause of disability in the United States, with mental and behavioral disorders in 2010 contributing to $13.6 \%$ of disability adjusted life years (DALYs), which is the sum of the years of life lost to premature mortality and the years lived with disability in a population, according to the World Health Organization (WHO). In the United States, the cost of lost earnings due to psychiatric diseases is estimated conservatively to be $\$ 200$ billion per year (Eaton et al. 2008). The burden of psychiatric diseases is particularly tragic because they typically begin early in life and are lifelong. For example, the onset of major psychiatric disorders such as schizophrenia, mood disorders, and substance use disorders is typically in late adolescence and young adulthood, often causing lifelong debilitation. Remarkably, major risk factors for such disorders may contribute to the pathology even in early development and adolescence. Therefore, understanding how disease-associated risk factors accumulate at earlier ages and eventually lead to the onset of disease in the developmental trajectory is crucial to advance the care of patients with these debilitating disorders.

Medical conditions in individuals are typically characterized by constellations of clinical signs and symptoms. They are reflections of pathological processes and mechanisms ( pathophysiology), which may arise from etiological factors, such as genetic variants or environmental insults (pathogenesis) (McHugh and Slavney 1998). Most major psychiatric disorders can also be conceptualized in a framework in which complex interactions of many genetic and environmental pathogenic factors converge onto common pathophysiological pathways, which in turn underlie the signs and symptoms of illness. Therefore, understanding the causal relationships among the pathogenesis, pathophysiology, and clinical phenotype is a critical goal in psychiatric research and an urgent issue for clinical objectives such as early diagnosis and intervention.

The high heritability of psychiatric disorders highlights a substantial contribution from genetic variants (Polderman et al. 2015). In the past decade, understanding the genetic architecture of psychiatric diseases has received much attention. Fueled by technological advances and collaborative efforts by large teams and consortia, psychiatric genetics has made robust and replicable genetic findings of common, rare, and de novo genetic variants (Sullivan et al. 2012). In particular, genome-wide association studies (GWASs) have successfully identified hundreds of common genetic variants associated with major psychiatric diseases such as schizophrenia (Schizophrenia Working Group of the Psychiatric Genomics 2014). However, direct translation of GWAS findings into disease mechanisms and new therapies is a fundamental challenge (Gandal et al. 2016). The current diagnostic criteria were formulated for clinical reliability and utility rather than biological validity, and as a result multiple conditions with heterogeneous etiologies are

(C) 2018 Namkung et al. This article is distributed under the terms of the Creative Commons Attribution-NonCommercial License, which permits reuse and redistribution, except for commercial purposes, provided that the original author and source are credited. 
included in each diagnostic category used by GWASs (such as schizophrenia or major depressive disorder). Deciphering how risk-conferring genetic variants with very small effect sizes individually contribute to clinical phenotypes is a great challenge. In particular, fine-mapping the "causal" variants that have biological impact is challenging because of the correlative structure of the genome, a phenomenon referred to as linkage disequilibrium (LD). Furthermore, given that the majority of disease-associated single-nucleotide polymorphisms (SNPs) are located in noncoding regions of the genome, underlying mechanisms are most likely regulatory, which makes it more challenging to elucidate the direct functional consequences of these variants. Acknowledging these limitations, in the post-GWAS era there is a critical need for new approaches that translate genetic findings into biological insights.

The goal of this review is, therefore, to construct a promising intellectual framework in which we may obtain causal, mechanistic insights into how multifactorial etiologies affect downstream biological processes that in turn drive phenotypes of psychiatric relevance. Keeping this goal in mind, we first give an overview of clinical nosology, which includes a contrast of the categorical and dimensional approaches. Although the classical categorical approach is still useful in clinical settings, the heterogeneity within each diagnostic category and pleiotropic impacts of genetic variants across multiple diseases challenge the biological validity of this approach, which may be complemented by the dimensional approach. We next introduce a framework that is helpful to delineate how pathogenic factors contribute to specific clinical symptoms or dimensions via pathophysiological pathways. We then present practical tools and approaches that apply clinical and scientific data onto this framework, aiming to translate genetic findings into biological insights. The method of expression quantitative trait loci (eQTL) mapping in conjunction with Mendelian randomization (MR) is presented as a tool to address causal inference from individual genetic variants to phenotypes of interest, whereas polygenic risk scoring (PRS) has been more recent- ly highlighted as a further systematic approach to integrate the aggregate effect of genetic variants with relevant phenotypes. We finally discuss promising human tissue/cell resources and the utility of animal models, through which clinical and preclinical studies can complement each other in a way that causally links genetic, molecular, cellular, circuitry, and behavioral levels.

\section{DIMENSIONAL AND CATEGORICAL APPROACH}

The diagnostic criteria formulated for psychiatric disorders reflect the aims of reliability and utility, by classifying signs and symptoms that differentiate one disorder from another (Owen 2014). Most clinicians use the Diagnostic and Statistical Manual of Mental Disorders (DSM) or the International Classification of Diseases (ICD) in their successive iterations, revised by the changing "consensus" of the field. The use of these phenotypic criteria to distinguish disorders from each other can be described as a categorical approach.

The dimensional approach seeks to view aspects of behavior and function as continuous distributions that are not constrained by current diagnostic categories or the occasionally unclear distinction between health and illness. For example, instead of viewing schizophrenia as a distinct and uniform entity, this approach considers each case as a combination of multiple dimensions, such as attention and working memory (Fig. 1). Every individual manifests a degree of attentional performance along a continuum, between levels that confer resilience in functioning and daily life, and levels that confer vulnerability and impairment, perhaps with a threshold level at one or both ends of the distribution that makes the dimension recognizable as an element of "disease." Furthermore, an individual's presentation of a certain dimension may shift along this continuum in response to environmental factors and lifestyle.

This framework is useful in studying behaviors that manifest with great heterogeneity within the population

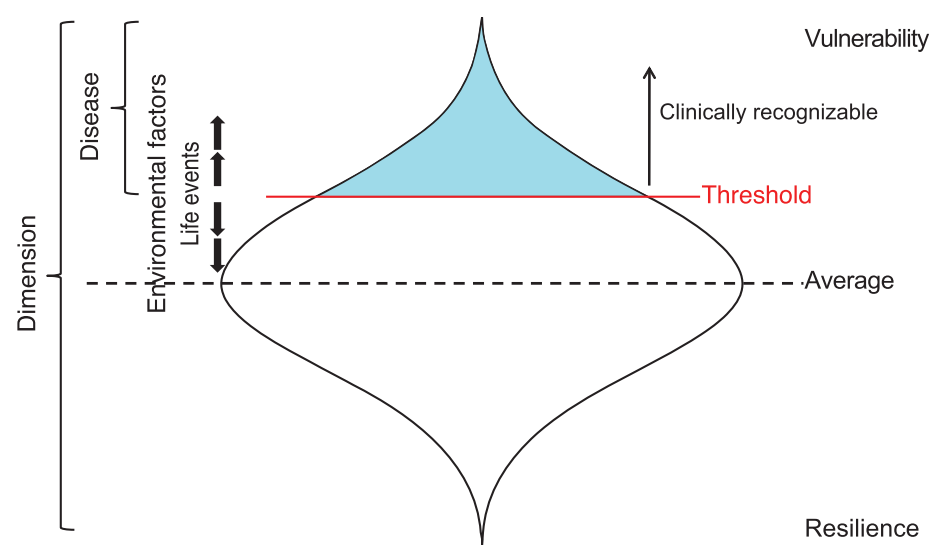

Figure 1. Modeling of brain disorders through a dimensional approach. Dimensions of behavior and function relevant to brain disorders may have a more continuous nature, with a distribution among the population. An individual may manifest this dimension somewhere along a continuum between levels that confer resilience versus vulnerability, with a threshold making the presentation recognizable as "disease" that can shift in response to environmental factors. 


\begin{tabular}{|c|c|c|c|}
\hline SZ1 & \multicolumn{3}{|l|}{$A B C$} \\
\hline SZ2 & \multicolumn{3}{|l|}{$A C D$} \\
\hline BP1 & \multicolumn{3}{|c|}{ C EFG } \\
\hline BP2 & \multicolumn{2}{|c|}{ DEF } & \\
\hline MDD1 & C & $\mathrm{GH}$ & \\
\hline MDD2 & D & $\mathrm{GHI}$ & \\
\hline SUD1 & C & IJ & \\
\hline SUD2 & $A$ & IJ & \\
\hline AD1 & C & $\mathrm{G}$ & $X Y Z$ \\
\hline AD2 & & G & $X Y Z$ \\
\hline
\end{tabular}

Figure 2. Cross-diagnostic dimensions in brain disorders. Brain disorders can be recognized as syndromes consisting of heterogeneous dimensions of behavior and function, many of which are shared among different disorders. (SZ) Schizophrenia, (BP) bipolar disorder, (MDD) major depressive disorder, (SUD) substance use disorder, (AD) Alzheimer's disease.

and the biological substrates (such as genes or neural circuits) underlying or impacting behavior that are likely to exert pleiotropic effects on other behaviors. In the context of psychiatric disorders, this approach addresses more specific aspects of disease and disability that are shared between multiple diagnostic categories to varying extents depending on the individual (Fig. 2). Thus, the study of certain dimensions may open new avenues of investigation into the relationships between these substrates and clinical phenotypes, and may lead to more mechanism-guided therapeutic strategies. Refining our understanding of these relationships may lead us to develop other diagnostic methods and define new clinical entities that better predict features such as course and outcome.

\section{MULTIPLE ETIOLOGIES (PATHOGENESIS) LEADING TO COMMON PATHWAYS (PATHOPHYSIOLOGY)}

To better understand the factors and processes underlying different dimensions in psychiatry, we may first distinguish those involved in pathogenesis or pathophysiology. For example, in disorders such as schizophrenia, there may be many pathogenic factors that contribute to aberrant development and dysfunction many years before the onset of symptoms, even as early as in utero, whereas pathophysiological factors can drive the development of symptoms from early adolescence and cause lifelong distress. The differences in causal effect and timing between these sets of factors have important implications, such as in designing therapeutics to intervene at different stages of the developmental trajectory.

This distinction is indeed very important in major psychiatric disorders, many of which are thought to be highly polygenic, indicated by GWASs, as well as highly pleiotropic, indicated by the significant overlap of risk variants between different disorders defined by the classical categorical approach. This genetic background interacts with environmental factors, both at the pathogenic level, shaping an individual's risk for the disorder, and at the level of downstream biological pathways linking these factors to the pathophysiology. The interaction of these pathogenic factors with each other and with pathophysiological processes is highlighted by emerging evidence of the increasing multitude and complexity of these factors in disorders such as schizophrenia and bipolar disorder.

For example, schizophrenia is thought to be a syndrome arising from developmental origins through an interplay of many risk factors that impact the development of many pathways and circuits in the brain over the course of illness (Fig. 3). In such complex disorders, it is plausible that many pathogenic factors converge to affect a smaller set

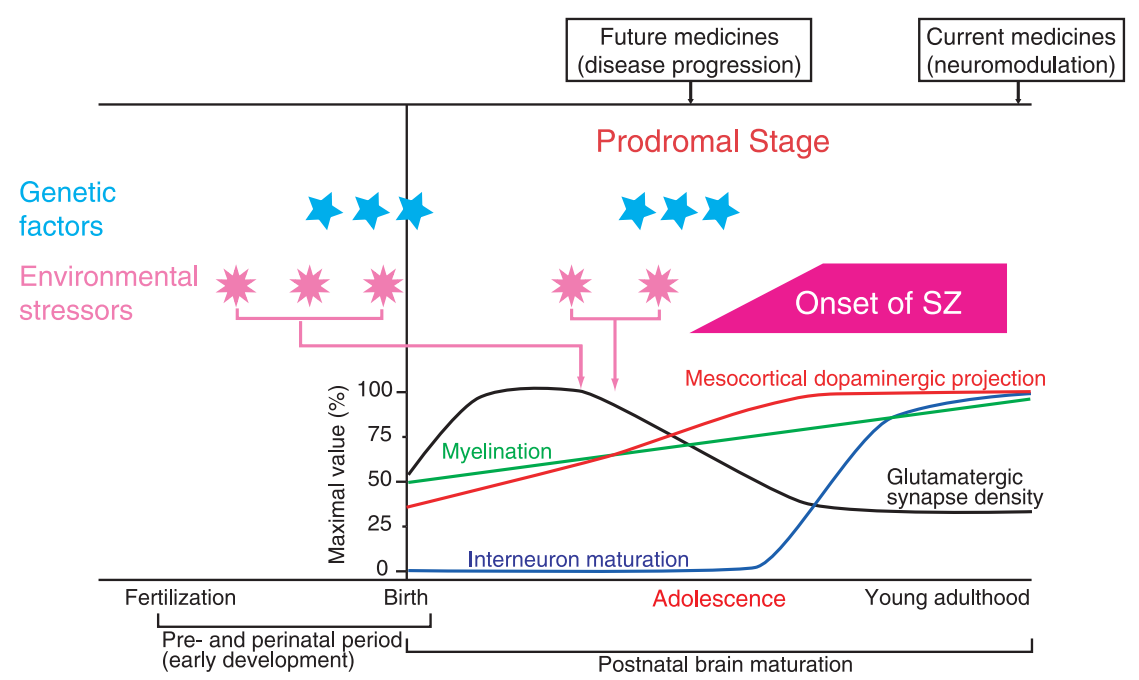

Figure 3. Developmental trajectory of molecular pathways in schizophrenia. Schizophrenia can be conceptualized as a global brain disorder, with genetic and environmental risk factors that shape the developmental trajectory of many molecular pathways. Treatment may target different stages, such as at the onset of symptoms or at earlier stages when it is possible to modify the course of illness. (Adapted, with permission, from Owen et al. 2016; originally adapted from Jaaro-Peled et al. 2009.) 


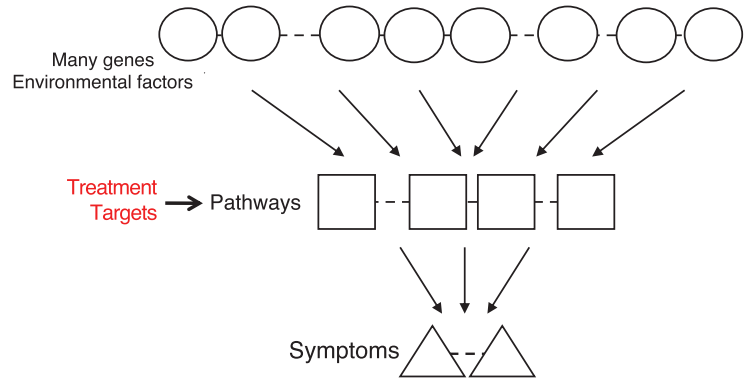

Figure 4. Model for convergence from pathogenic factors to pathophysiological pathways to symptoms. There is evidence that the multitude of genetic and environmental risk factors in the pathogenesis of major psychiatric disorders converge onto a smaller number of pathophysiological pathways, which in turn converge onto specific symptoms or dimensions. A better understanding of the pathophysiological processes that link pathogenic factors to clinical phenotypes may yield novel therapeutic targets that improve on current treatments that target symptoms. (Adapted, with permission, from Srivastava et al. 2018.)

of pathophysiological pathways (Fig. 4), for which largescale genomic studies have begun to yield evidence (Owen 2014; Owen et al. 2016). These sites of convergence may provide novel therapeutic targets that intervene at the level of pathophysiology, which may be more tractable than the level of pathogenesis, especially for disorders with complex genetic architectures and gene-environment interactions, or the level of symptoms, which may not be adequate to alter disease course and improve functional outcomes or quality of life (Jaaro-Peled et al. 2009; Landek-Salgado et al. 2016).

Current treatments for psychiatric disorders mostly control symptoms. However, with improved understanding of the pathophysiological pathways underlying each symptom, and the pathogenic factors that influence each pathway, it may be possible to develop novel strategies to alter the course of a patient's illness to achieve better outcomes (Figs. 3 and 4). Indeed, targeting causal mediators that receive converging influence from pathogenic factors may be a more efficient way to treat disorders with complex pathogenesis and heterogeneity. Furthermore, identifying pathophysiological factors in the early stages of disease that underlie specific dimensions may allow earlier detection or even prediction of specific symptoms, which is important for tailoring treatment of disorders with long and complex developmental trajectories.

\section{TRANSLATING GENETIC FINDINGS INTO DISEASE MECHANISMS}

The advances in -omics profiling have helped devise strategies for interrogating pathogenic factors, such as common genetic variants and environmental stressors, with intermediate pathophysiological mediators, such as transcripts, proteins, or metabolites. One such strategy has been expression quantitative trait loci (eQTL) mapping, which identifies individual variants that are associated with changes in gene expression in a given tissue or cell type (Albert and Kruglyak 2015). The colocalization of eQTLs and GWAS hits enables us to prioritize potentially causal genetic variants that may underlie diseaseassociated phenotypes through gene expression regulation. A recent study using brain eQTL data sets (Fromer et al. 2016), for example, has elucidated genetic variants in 20 of 108 schizophrenia GWAS risk loci, identified by the Psychiatric Genomics Consortium phase 2 (PGC2) study, that alter expression of one or more genes in the dorsolateral prefrontal cortex (DLPFC). Several of the genes were further validated by experimental manipulations and shown to affect neuroanatomical and developmental attributes in model systems, making these genes potential candidates for further biological investigation. As such, eQTL mapping, in conjunction with experimental validation, serves as a promising strategy to integrate potentially causal genetic variants with their downstream biological processes.

Although considerable effort in integrating GWAS and eQTL data has been made to identify genes whose expression is significantly associated with particular traits, they do not aim to estimate the strength of the causal effect and are unable to distinguish causation from pleiotropy (i.e., when a genetic variant influences multiple phenotypes). One of the most widely appreciated approaches to address such causal effect of gene expression on phenotypes has been MR (Smith and Ebrahim 2003; Lawlor et al. 2008), in which a genetic variant (e.g., a SNP) is used as an instrumental variable (IV) to test for the causative effect of an exposure (e.g., gene expression) on an outcome (phenotype). For example, a recent Mendelian randomization (MR) study using a SNP in the interleukin-6 receptor (IL-6R) gene as an IV has suggested that the signaling involving IL-6R as an exposure may have a causal role in the development of coronary heart disease as an outcome (Interleukin-6 Receptor Mendelian Randomisation Analysis et al. 2012).

A number of advantages render MR popular, including the capability to control for nonheritable environmental confounders and to evaluate the impact of an exposure without necessitating the measurement of that exposure in the outcome group (Smith and Ebrahim 2004). However, a fundamental assumption of MR is "no horizontal pleiotropy," which means that genetic variants used as the IV cannot have pleiotropic effects (Burgess et al. 2018). Violation of the "no horizontal pleiotropy" assumption occurs when the genetic variant influences the outcome through pathways independent of the exposure of interest. Once violated, MR tests can be severely distorted, leading to inaccurate causal estimates, loss of statistical power, and potential false-positive causal relationships. Therefore, considering the pervasive pleiotropy amongst disease-associated variants identified by GWASs, some have raised skepticism about the utility of MR in complex diseases (Pickrell 2015). Indeed, recent insights into psychiatric genetics have shown that many traits are genetically correlated with each other (Bulik-Sullivan et al. 2015); plus hundreds of genetic variants identified by GWASs are associated with multiple traits (Sivakumaran et al. 2011; Gratten and Visscher 2016). Therefore, caution should be taken in conducting MR, such as performing a systematic evaluation and correction for horizontal pleiotropic outli- 
ers (Verbanck et al. 2018). In parallel, a more suitable approach for causal inference from genetic variants (in polygenic and pleiotropic architectures) to phenotypes through an intermediate pathway would be greatly beneficial.

Although eQTL mapping in conjunction with MR provides a microscopic approach for causal inference from individual genetic variants to phenotypes of interest, capturing the aggregate effect of genetic variants into a single value representing an individual's overall genetic risk and integrating it with downstream biological pathways can provide another promising strategy at the macroscopic and systematic level, given the highly polygenic architecture of psychiatric diseases. The polygenic risk score (PRS) of an individual can be a good measure to summarize the aggregate effect of pathogenic genetic variants, typically calculated as the sum of disease-associated alleles across the genome weighted by effect size (Chatterjee et al. 2016). Thus, the PRS provides a continuous, quantitative measure of an individual's overall genetic burden that can be associated with pathophysiological measures, such as gene/protein expression and brain activity/connectivity. For instance, PRSs have been recently associated with functional/structural brain imaging measures as well as cognitive performance in psychosis (Ranlund et al. 2018). Likewise, the quantification of PRSs coupled with intermediate pathophysiological mediators can facilitate the translation of genetic findings into biological insights. However, considerable efforts should be undertaken to improve the utility of PRSs. Given that PRSs are population-specific (Martin et al. 2017), there is a great need to expand our efforts to more diverse populations, including those of African, Hispanic, and Asian descent, so that individuals within these populations can benefit from the promise of genetic advances. Furthermore, beyond the current method of quantifying PRSs that reflects only common genetic burden, a much more comprehensive quantification method should be developed to encompass overall genetic risks (i.e., common, intermediate, and rare genetic variants) as well as environmental risks, given the multifactorial etiologies of psychiatric diseases.

\section{TOWARD CAUSAL, MECHANISTIC INSIGHTS}

Identifying pathophysiological molecular pathways associated with psychiatric diseases is crucial in building a mechanistic bridge between pathogenesis, pathophysiology, and clinical phenotypes. Thus, access to neural tissues and cells that reflect disease-associated molecular phenotypes is important. Although postmortem brains have been appreciated as informative resources, they do not reliably provide molecular signatures associated with the onset or the course of functional impairments in living patients (Lavoie et al. 2017a,b). The engineered cell models derived from living humans via tissue biopsy, including induced neuronal (iN) cells and induced pluripotent stem cell (iPSC)-derived neurons, can also be useful since they may reflect neuronal "traits" of brain cells. However, their molecular and cellular signatures at the time of biopsy ("state" change) may be lost in the process of cell reprogramming and conversion. To complement the limitations in postmortem brains and engineered cell models, surrogate tissues that capture both neuronal "states" and "traits" directly correlated with clinical symptoms are needed. At present, olfactory epithelium-derived neuronal cells may serve as at least one useful surrogate model to capture both dynamic changes ("states") and persistent signatures ("traits") that are directly associated with psychiatric phenotypes at the molecular level (Fig. 5). Therefore, constructing an approach that enables causal inference from multifactorial etiologies to dimensions of psychiatric relevance, through molecular pathways captured by olfactory neuronal cells, can be promising in obtaining a comprehensive mechanistic picture.

Animal studies provide a great opportunity to obtain sophisticated, causal insights into psychiatric diseases by back-translating observations from human data (Namkung et al. 2017). Once a key molecular pathway is identified in humans, a relevant rodent model that best reflects the human findings can be generated. Then, causal insights at multiple levels, spanning from molecules, cells, circuitry, to behavior, can be obtained by conducting invasive brain manipulations, which are critical to address causation and present major ethical constraints in human studies. The activity of anatomically and genetically defined neural cells and circuits (Lerner et al. 2016) can be monitored and manipulated in behaviorally relevant contexts in the rodent model, using miniature microscopes for activity readouts (Ziv and Ghosh 2015) and optogenetic/ chemogenetic approaches for activity manipulations (Deisseroth 2011; Urban and Roth 2015). In addition to rodents, nonhuman primates such as marmosets are a highly valid model system, especially when investigating higher cognitive and social functions that may not be well conserved in rodents.

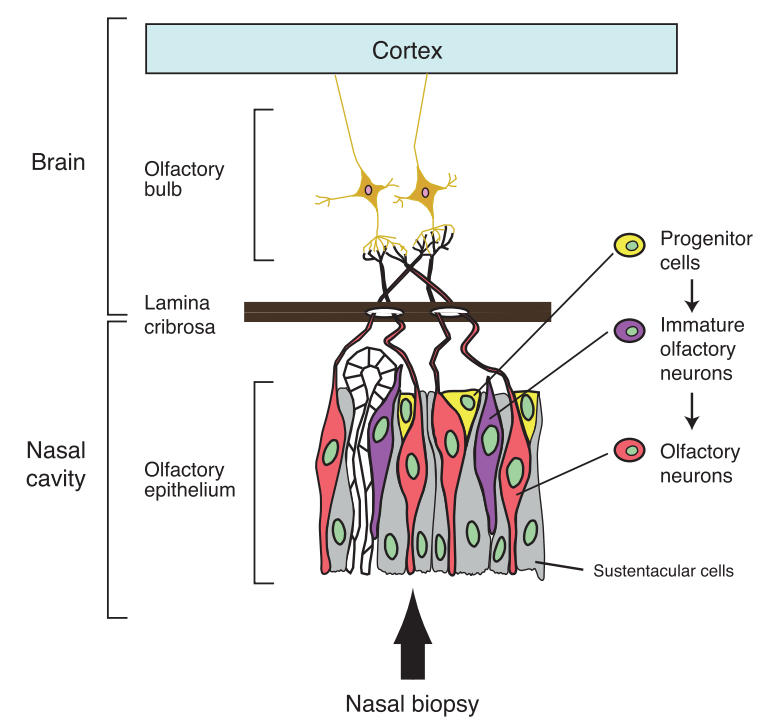

Figure 5. The human olfactory epithelium with neural progenitors. The olfactory neural epithelium is composed of different cell types including basal cells (progenitor cells), olfactory neurons (mature and immature), and sustentacular cells. (Adapted, with permission, from Lavoie et al. 2017a.) 


\section{CONCLUSION}

We have sought to present an intellectual framework in which we may obtain causal, mechanistic insights into how multifactorial etiologies affect downstream biological processes that in turn drive clinical symptoms or dimensions of psychiatric relevance. For this purpose, we introduced a foundation of clinical nosology, the categorical versus dimensional approaches. We then introduced a framework to delineate how pathogenic factors contribute to specific clinical symptoms or dimensions via pathophysiological pathways. Several tools and approaches useful for translating genetic findings into biological insights, such as eQTL, MR, and PRS, were presented. Promising human tissue/cell resources and the utility of animal models were also proposed. Taken together, we propose that this intellectual framework allows clinical and preclinical studies to complement each other in a way that causally links genetic, molecular, cellular, circuitry, and behavioral levels.

\section{ACKNOWLEDGMENTS}

We thank Yukiko Lema for preparing the figures, and Sedona Lockhart for proofreading the manuscript. This study was supported by the National Institute of Mental Health (MH-092443, MH-094268, MH-105660, and MH107730) and foundation grants from Stanley and S-R/ RUSK, and Brains and Behavior Research Foundation (BBRF).

\section{REFERENCES}

Albert FW, Kruglyak L. 2015. The role of regulatory variation in complex traits and disease. Nat Rev Genet 16: 197-212. doi:10 $.1038 / \operatorname{nrg} 3891$

Bulik-Sullivan B, Finucane HK, Anttila V, Gusev A, Day FR, Loh PR, ReproGen Consortium, Psychiatric Genomics Consortium; Genetic Consortium for Anorexia Nervosa of the Wellcome Trust Case Control Consortium 3, Duncan L, et al. 2015. An atlas of genetic correlations across human diseases and traits. Nat Genet 47: 1236-1241. doi:10.1038/ ng.3406

Burgess S, Foley CN, Zuber V. 2018. Inferring causal relationships between risk factors and outcomes from genome-wide association study data. Annu Rev Genomics Hum Genet 19: 303-327. doi:10.1146/annurev-genom-083117-021731

Chatterjee N, Shi J, García-Closas M. 2016. Developing and evaluating polygenic risk prediction models for stratified disease prevention. Nat Rev Genet 17: 392-406. doi:10.1038/nrg .2016 .27

Deisseroth K. 2011. Optogenetics. Nat Methods 8: 26-29. doi:10 .1038/nmeth.f.324

Eaton WW, Martins SS, Nestadt G, Bienvenu OJ, Clarke D, Alexandre P. 2008. The burden of mental disorders. Epidemiol Rev 30: 1-14. doi:10.1093/epirev/mxn011

Fromer M, Roussos P, Sieberts SK, Johnson JS, Kavanagh DH, Perumal TM, Ruderfer DM, Oh EC, Topol A, Shah HR, et al. 2016. Gene expression elucidates functional impact of polygenic risk for schizophrenia. Nat Neurosci 19: 1442-1453. doi: $10.1038 / \mathrm{nn} .4399$

Gandal MJ, Leppa V, Won H, Parikshak NN, Geschwind DH. 2016. The road to precision psychiatry: translating genetics into disease mechanisms. Nat Neurosci 19: 1397-1407. doi:10.1038/nn.4409
Gratten J, Visscher PM. 2016. Genetic pleiotropy in complex traits and diseases: implications for genomic medicine. Genome Med 8: 78. doi:10.1186/s13073-016-0332-x

Interleukin-6 Receptor Mendelian Randomisation Analysis Consortium, Swerdlow DI, Holmes MV, Kuchenbaecker KB, Engmann JE, Shah T, Sofat R, Guo Y, Chung C, Peasey A, et al. 2012. The interleukin-6 receptor as a target for prevention of coronary heart disease: a mendelian randomisation analysis. Lancet 379: 1214-1224. doi:10.1016/S0140-6736(12) 60110-X

Jaaro-Peled H, Hayashi-Takagi A, Seshadri S, Kamiya A, Brandon NJ, Sawa A. 2009. Neurodevelopmental mechanisms of schizophrenia: understanding disturbed postnatal brain maturation through neuregulin-1-ErbB4 and DISC1. Trends Neurosci 32: 485-495. doi:10.1016/j.tins.2009.05.007

Landek-Salgado MA, Faust TE, Sawa A. 2016. Molecular substrates of schizophrenia: homeostatic signaling to connectivity. Mol Psychiatry 21: 10-28. doi:10.1038/mp.2015.141

Lavoie J, Gassó Astorga P, Segal-Gavish H, Wu YC, Chung Y, Cascella NG, Sawa A, Ishizuka K. 2017a. The olfactory neural epithelium as a tool in neuroscience. Trends Mol Med 23: 100103. doi:10.1016/j.molmed.2016.12.010

Lavoie J, Sawa A, Ishizuka K. 2017b. Application of olfactory tissue and its neural progenitors to schizophrenia and psychiatric research. Curr Opin Psychiatry 30: 176-183. doi:10 $.1097 /$ YCO.0000000000000327

Lawlor DA, Harbord RM, Sterne JA, Timpson N, Davey Smith G. 2008. Mendelian randomization: using genes as instruments for making causal inferences in epidemiology. Stat Med 27: 1133-1163. doi:10.1002/sim.3034

Lerner TN, Ye L, Deisseroth K. 2016. Communication in neural circuits: tools, opportunities, and challenges. Cell 164: 11361150. doi:10.1016/j.cell.2016.02.027

Martin AR, Gignoux CR, Walters RK, Wojcik GL, Neale BM, Gravel S, Daly MJ, Bustamante CD, Kenny EE. 2017. Human demographic history impacts genetic risk prediction across diverse populations. Am J Hum Genet 100: 635-649. doi:10 .1016/j.ajhg.2017.03.004

McHugh PR, Slavney PR. 1998. The perspectives of psychiatry, 2nd ed. The Johns Hopkins University Press, Baltimore.

Namkung H, Kim SH, Sawa A. 2017. The insula: an underestimated brain area in clinical neuroscience, psychiatry, and neurology. Trends Neurosci 40: 200-207. doi:10.1016/j.tins.2017 .02 .002

Owen MJ. 2014. New approaches to psychiatric diagnostic classification. Neuron 84: 564-571. doi:10.1016/j.neuron.2014 .10 .028

Owen MJ, Sawa A, Mortensen PB. 2016. Schizophrenia. Lancet 388: 86-97. doi:10.1016/S0140-6736(15)01121-6

Pickrell J. 2015. Fulfilling the promise of Mendelian randomization. bioRxiv doi:10.1101/018150

Polderman TJC, Benyamin B, de Leeuw CA, Sullivan PF, van Bochoven A, Visscher PM, Posthuma D. 2015. Metaanalysis of the heritability of human traits based on fifty years of twin studies. Nat Genet 47: 702-709. doi:10.1038/ ng.3285

Ranlund S, Calafato S, Thygesen JH, Lin K, Cahn W, CrespoFacorro B, de Zwarte SMC, Díez Á, Di Forti M, et al. 2018. A polygenic risk score analysis of psychosis endophenotypes across brain functional, structural, and cognitive domains. Am J Med Genet B Neuropsychiatr Genet 177: 21-34. doi:10.1002/ajmg.b.32581

Schizophrenia Working Group of the Psychiatric Genomics Consortium. 2014. Biological insights from 108 schizophreniaassociated genetic loci. Nature 511: 421-427. doi:10.1038/ nature 13595

Sivakumaran S, Agakov F, Theodoratou E, Prendergast JG, Zgaga L, Manolio T, Rudan I, McKeigue P, Wilson JF, Campbell H. 2011. Abundant pleiotropy in human complex diseases and traits. Am J Hum Genet 89: 607-618. doi:10.1016/j.ajhg .2011.10.004

Smith GD, Ebrahim S. 2003. 'Mendelian randomization': can genetic epidemiology contribute to understanding environ- 


\section{CAUSAL INFERENCE ON PATHOPHYSIOLOGICAL MEDIATORS}

mental determinants of disease? Int $J$ Epidemiol 32: 1-22. doi:10.1093/ije/dyg070

Smith GD, Ebrahim S. 2004. Mendelian randomization: prospects, potentials, and limitations. Int J Epidemiol 33: 30-42. doi:10.1093/ije/dyh132

Srivastava R, Faust T, Ramos A, Ishizuka K, Sawa A. 2018. Dynamic changes of the mitochondria in psychiatric illnesses: new mechanistic insights from human neuronal models. Biol Psychiatry 83: 751-760. doi:10.1016/j.biopsych.2018.01.007 Sullivan PF, Daly MJ, O'Donovan M. 2012. Genetic architectures of psychiatric disorders: the emerging picture and its implications. Nat Rev Genet 13: 537-551. doi:10.1038/nrg3240
Urban DJ, Roth BL. 2015. DREADDs (Designer Receptors Exclusively Activated by Designer Drugs): chemogenetic tools with therapeutic utility. Annu Rev Pharmacol 55: 399-417. doi:10.1146/annurev-pharmtox-010814-124803

Verbanck M, Chen CY, Neale B, Do R. 2018. Detection of widespread horizontal pleiotropy in causal relationships inferred from Mendelian randomization between complex traits and diseases. Nat Genet 50: 693-698. doi:10.1038/s41588-0180099-7

Ziv Y, Ghosh KK. 2015. Miniature microscopes for large-scale imaging of neuronal activity in freely behaving rodents. Curr Opin Neurobiol 32: 141-147. doi:10.1016/j.conb.2015.04.001 


\section{$\$_{\text {CSH }}^{\infty}$ Cold Spring Harbor Symposia SYMPOSIA On Quantitative Biology}

\section{Causal Inference on Pathophysiological Mediators in Psychiatry}

Ho Namkung, Brian J. Lee and Akira Sawa

Cold Spring Harb Symp Quant Biol 2018 83: 17-23 originally published online March 8, 2019 Access the most recent version at doi:10.1101/sqb.2018.83.037655

References This article cites 32 articles, 1 of which can be accessed free at: http://symposium.cshlp.org/content/83/17.full.html\#ref-list-1

Creative This article is distributed under the terms of the

Commons http://creativecommons.org/licenses/by-nc/4.0/, which permits reuse and License redistribution, except for commercial purposes, provided that the original author and source are credited.

Email Alerting Receive free email alerts when new articles cite this article - sign up in Service the box at the top right corner of the article or click here. 